\title{
Hard exclusive pion leptoproduction
}

Received: date / Accepted: date

\begin{abstract}
In this talk it is reported on an analysis of hard exclusive leptoproduction of pions within the handbag approach. It is argued that recent measurements of this process performed by HERMES and CLAS clearly indicate the occurrence of strong contributions from transversely polarized photons. Within the handbag approach such $\gamma_{T}^{*} \rightarrow \pi$ transitions are described by the transversity GPDs accompanied by twist-3 pion wave functions. It is shown that the handbag approach leads to results on cross sections and single-spin asymmetries in fair agreement with experiment. Predictions for other pseudoscalar meson channels are also briefly discussed.
\end{abstract}

Keywords Pion production · factorization · twist- $3 \cdot$ transversity

\section{Introduction}

The handbag approach to hard exclusive leptoproduction of mesons offers a partonic description of these processes in the generalized Bjorken regime of large photon virtuality, $Q^{2}$, and large photonproton center of mass energy, $W$, but small invariant momentum transfer, $-t$. The theoretical basis of the handbag approach is provided by the factorization theorems [1,2] which say that the process amplitudes for longitudinally polarized virtual photons, $\gamma_{L}^{*}$, are represented as convolutions of hard partonic subprocess amplitudes and soft hadronic matrix elements, so-called generalized parton distributions (GPDs), which encode the soft physics. The subprocess amplitudes are likewise convolutions of perturbatively calculable hard scattering kernels and meson wave functions. For pion production in particular the helicity amplitudes for $\gamma_{L}^{*} \rightarrow \pi$ transitions read

$$
\mathcal{M}_{0+, 0+}=\frac{e_{0}}{Q} \sqrt{1-\xi^{2}}\left[\langle\tilde{H}\rangle-\frac{\xi^{2}}{1-\xi^{2}}\langle\tilde{E}\rangle\right], \quad \mathcal{M}_{0-, 0+}=\frac{e_{0}}{Q} \frac{\sqrt{-t^{\prime}}}{2 m} \xi\langle\tilde{E}\rangle,
$$

where

$$
\langle K\rangle=\int_{-1}^{1} d x \sum_{\lambda} \mathcal{H}_{0 \lambda, 0 \lambda}\left(x, \xi, Q^{2}\right) K(x, \xi, t)
$$

denotes the convolution of a subprocess amplitude, $\mathcal{H}$, with a GPD $K$. The nucleon mass is denoted by $m$. The skewness, $\xi$, is related to Bjorken-x by $\xi=x_{B} /\left(1-x_{B}\right)$ up to corrections of order $1 / Q^{2}$. In (1) the usual abbreviation $t^{\prime}=t-t_{0}$ is employed where the minimal value of $-t$ corresponding to forward scattering, is $t_{0}=-4 m^{2} \xi^{2} /\left(1-\xi^{2}\right)$. Helicities are labeled by their signs or by zero; they appear in the familiar order: pion, outgoing nucleon, photon, in-going nucleon.

P. Kroll

Fachbereich Physik, Universität Wuppertal, 42097 Wuppertal

Tel.: +49-202-4392620

Fax: +49-202-4393811

E-mail: pkroll@uni-wuppertal.de 
Power corrections to the leading-twist result (11) are theoretically not under control. It is therefore not clear at which values of $Q^{2}$ and $W$ the amplitudes (11) can be applied. The onset of the leading-twist dominance has to be found out by comparison with experiment. An example of power corrections is set by the amplitudes for transversely polarized photons, $\gamma_{T}^{*}$, which are suppressed by $1 / Q$ as compared to the $\gamma_{L}^{*} \rightarrow \pi$ amplitudes. Still, as experiments tell us, the $\gamma_{T}^{*} \rightarrow \pi$ amplitudes play an important role in hard exclusive pion leptoproduction. The first experimental evidence for strong contribution from $\gamma_{T}^{*} \rightarrow \pi$ transitions came from the spin asymmetry, $A_{U T}$, measured with a transversely polarized target by the HERMES collaboration for $\pi^{+}$production [3. Its $\sin \phi_{s}$ modulation 1 unveils a particularly striking behavior: It is rather large and does not show any indication of a turnover towards zero for $t^{\prime} \rightarrow 0$. In this limit $A_{U T}^{\sin \phi_{s}}$ is in control of an interference term of two helicity non-flip amplitudes

$$
A_{U T}^{\sin \phi_{s}} \propto \operatorname{Im}\left[\mathcal{M}_{0-,++}^{*} \mathcal{M}_{0+, 0+}\right] .
$$

Both the amplitudes are not forced by angular momentum conservation to vanish in the limit $t^{\prime} \rightarrow 0$. Hence, the small $-t^{\prime}$ behavior of $A_{U T}^{\sin \phi_{s}}$ entails a sizeable $\gamma_{T}^{+} \rightarrow \pi$ amplitude.

A second evidence for large contributions from transversely polarized photons comes from the CLAS measurement [4 of the $\pi^{0}$ electroproduction cross sections. As can be seen from Fig. 1] the transverse-transverse interference cross section is negative and amounts to about $50 \%$ of the unseparated cross section in absolute value. Neglecting the double-flip amplitude $\mathcal{M}_{0-,-+}\left(\propto t^{\prime}\right.$ for $\left.t^{\prime} \rightarrow 0\right)$ and introducing the combinations

$$
\mathcal{M}_{0+, \mu+}^{N, U}=\frac{1}{2}\left[\mathcal{M}_{0+, \mu+} \pm \mathcal{M}_{0+,-\mu+}\right]
$$

one can write the transverse-transverse interference cross section as

$$
\frac{d \sigma_{T T}}{d t} \simeq-\frac{1}{\kappa}\left[\left|\mathcal{M}_{0+,++}^{N}\right|^{2}-\left|\mathcal{M}_{0+,++}^{U}\right|^{2}\right]
$$

where $\kappa$ is a phase-space factor. Thus, from the CLAS data one learns that $\left|\mathcal{M}_{0+,++}^{N}\right| \gg\left|\mathcal{M}_{0+,++}^{U}\right|$ and is also large in comparison to the amplitudes (1).

In passing I remark that the combinations (4) are special cases of natural $(N)$ and unnatural parity $(U)$ combinations. They satisfy the symmetry relations

$$
\mathcal{M}_{0 \nu^{\prime},-\mu \nu}^{N, U}=\mp(-1)^{\mu} \mathcal{M}_{0 \nu^{\prime}, \mu \nu}^{N, U}
$$

as a consequence of parity conservation 2 .

\section{Transversity}

In Fig. 2 a typical Feynman graph for pion production is depicted with helicity labels for the amplitude $\mathcal{M}_{0-,++}$. Angular momentum conservation forces both the subprocess amplitude and the nucleonparton matrix element to vanish $\propto \sqrt{-t^{\prime}}$ for $t^{\prime} \rightarrow 0$ for any contribution to $\mathcal{M}_{0-,++}$ from the usual helicity non-flip GPDs $\widetilde{H}$ and $\tilde{E}$. This result is in conflict with the HERMES data on $A_{U T}^{\sin \phi_{s}}$. However, there is a second set of GPDs, the helicity-flip or transversity ones, $H_{T}, \widetilde{H}_{T}, E_{T}, \tilde{E}_{T}$ [6] for which the emitted and reabsorbed partons have opposite helicities. In [5] (see also [9]), it has been suggested that contributions from the transversity GPDs are responsible for the above mentioned experimental phenomena. Assuming handbag factorization for the $\gamma_{T}^{*} \rightarrow \pi$ amplitudes, they read $(\mu= \pm 1)$

$$
\begin{aligned}
& M_{0+\mu+}=e_{0} \frac{\sqrt{-t^{\prime}}}{4 m} \int_{-1}^{1} d x\left\{\left(\mathcal{H}_{0+\mu-}-\mathcal{H}_{0-\mu+}\right)\left(\bar{E}_{T}-\xi \widetilde{E}_{T}\right)\right. \\
&\left.+\left(\mathcal{H}_{0+\mu-}+\mathcal{H}_{0-\mu+}\right)\left(\widetilde{E}_{T}-\xi E_{T}\right)\right\}
\end{aligned}
$$

\footnotetext{
1 The angle $\phi_{s}$ defines the orientation of the target spin vector.

2 Parity conservation leads to the relation $\mathcal{M}_{0-\nu^{\prime},-\mu-\nu}=-(-1)^{\mu-\nu+\nu^{\prime}} \mathcal{M}_{0 \nu^{\prime}, \mu \nu}$. An analogous relation holds for the subprocess amplitudes.
} 

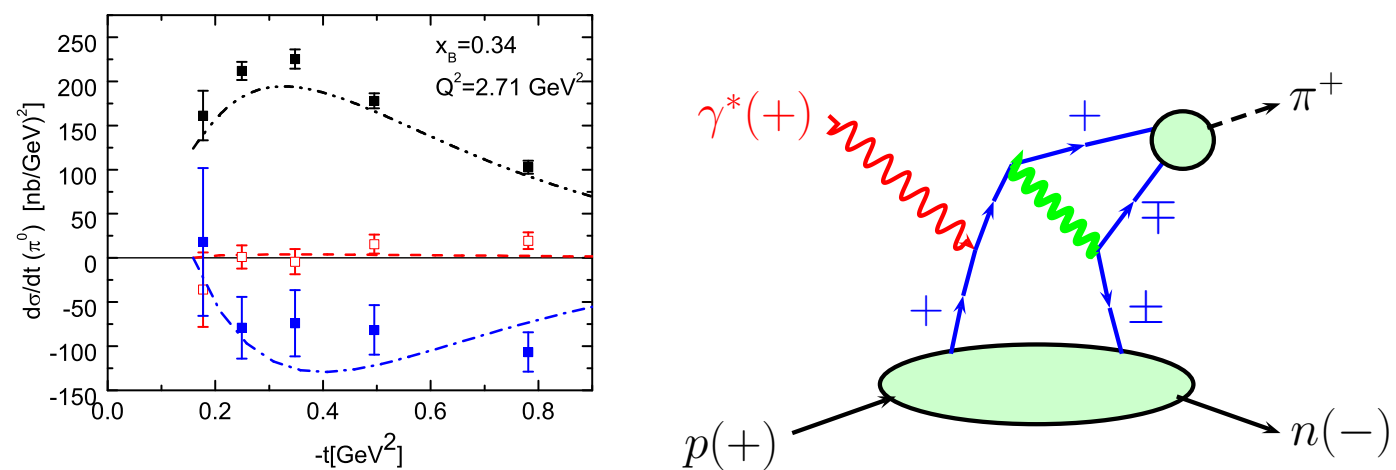

Fig. 1 The unseparated (long.-transv., transv.-transv.) cross section. Data 4 are shown as solid (open, solid) symbols. The theoretical results are taken from [5].

Fig. 2 A typical lowest-order Feynman graph for pion leptoproduction. The signs indicate the helicities of the involved particles.

$$
\begin{aligned}
M_{0-\mu+}=e_{0} \sqrt{1-\xi^{2}} \int_{-1}^{1} d x & \left\{\mathcal{H}_{0-\mu+}\left[H_{T}+\frac{\xi}{1-\xi^{2}}\left(\widetilde{E}_{T}-\xi E_{T}\right)\right]\right. \\
& \left.+\left(\mathcal{H}_{0+\mu-}-\mathcal{H}_{0-\mu+}\right) \frac{t^{\prime}}{4 m^{2}} \widetilde{H}_{T}\right\} .
\end{aligned}
$$

With the help of parity conservation one can easily convince oneself that the nucleon helicity non-flip amplitude consists of a natural and a unnatural parity part. The natural parity part is related to the GPD $\bar{E}_{T} \equiv 2 \widetilde{H}_{T}+E_{T}$ with corrections 3 of order $\xi^{2}$ from $\tilde{E}_{T}$. The unnatural part is proportional to $\xi$. The amplitude $M_{0-\mu+}$ is more complicated. There is a contribution that does not have a definite parity behavior. It is dominated by $H_{T}$ with corrections of order $\xi^{2}$ from $E_{T}$ and $\widetilde{E}_{T}$. It contributes to $M_{0-++}$ while its contribution to $M_{0--+}$ is suppressed by $t / Q^{2}$ from the double-flip subprocess amplitude $H_{0-,-+}$. In addition there is a natural parity contribution to $\mathcal{M}_{0-, \mu+}$ from $\widetilde{H}_{T}$ which is suppressed by $t^{\prime} /\left(4 m^{2}\right)$. Thus, the $\gamma_{T}^{*} \rightarrow \pi$ amplitudes advocated for in [5, 8 ]

$$
\begin{aligned}
& \mathcal{M}_{0+, \mu+}=e_{0} \frac{\sqrt{-t^{\prime}}}{4 m} \int_{-1}^{1} d x \mathcal{H}_{0-,++} \bar{E}_{T} \\
& \mathcal{M}_{0-,++}=e_{0} \sqrt{1-\xi^{2}} \int_{-1}^{1} d x \mathcal{H}_{0-,++} H_{T} \\
& \mathcal{M}_{0-,-+}=0
\end{aligned}
$$

are justified within the handbag approach at least at small $\xi$ and $-t^{\prime}$. There is only one subprocess amplitude common to the transverse amplitudes. These amplitudes meet the main features of the experimental data discussed in Sect. 1.

\section{The subprocess amplitude $\boldsymbol{H}_{0-,++}$}

As can be seen from Fig. 2 quark and antiquark forming the pion have the same helicity. Hence, a twist-3 pion wave function is required. There are two twist-3 distribution amplitudes, a pseudoscalar one, $\Phi_{P}$ and a pseudotensor one, $\Phi_{\sigma}$. Assuming the three-particle contributions to be strictly zero, one obtains the twist-3 distribution amplitudes [10]

$$
\Phi_{P} \equiv 1 \quad \Phi_{\sigma}=6 \tau(1-\tau)
$$

from the equation of motion. Here $\tau$ is the momentum fraction the quark in the pion carries with respect to the pion momentum. The subprocess amplitude $\mathcal{H}_{0-,++}$ is computed to lowest-order of

\footnotetext{
3 The GPD $\tilde{E}_{T}$ is an odd function of $\xi$ as a consequence of time reversal invariance [7].
} 
perturbation theory (a typical Feynman graph is shown in Fig. 22). It turns out that the pseudotensor term is proportional to $t / Q^{2}$ and consequently neglected. The pseudoscalar term is non-zero at $t=0$ but infrared singular. In order to regularize this singularity the modified perturbative approach is used in 5. 8 in which quark transverse momentum, $k_{\perp}$, are retained in the subprocess while the emission and reabsorption of partons from the nucleons is assumed to happen collinear to the nucleon momenta [11. In this approach the subprocess amplitude reads

$$
\begin{aligned}
\mathcal{H}_{0-,++}= & \frac{2}{\pi^{2}} \frac{C_{F}}{\sqrt{2 N_{C}}} \mu_{\pi} \int d \tau d^{2} \mathbf{k}_{\perp} \Psi_{\pi P}\left(\tau, k_{\perp}\right) \alpha_{s}\left(\mu_{R}\right) \\
\times & {\left[\frac{e_{u}}{x-\xi+i \epsilon} \frac{1}{\bar{\tau} \frac{x-\xi}{2 \xi} Q^{2}-k_{\perp}^{2}+i \epsilon}\right.} \\
& \left.-\frac{e_{d}}{x+\xi-i \epsilon} \frac{1}{-\tau \frac{x+\xi}{2 \xi} Q^{2}-k_{\perp}^{2}+i \epsilon}\right] .
\end{aligned}
$$

Here $4, \Psi_{\pi P}$ is a light-cone wave function for the pion which is parametrized as 5

$$
\Psi_{\pi P}=\frac{16 \pi^{3 / 2}}{\sqrt{2 N_{C}}} f_{\pi} a_{P}^{3}\left|\mathbf{k}_{\perp}\right| \exp \left[-a_{P}^{2} k_{\perp}^{2}\right] .
$$

Its associated distribution amplitude is the pseudoscalar one given in (9). For the transverse-size parameter, $a_{P}$, the value $1.8 \mathrm{GeV}$ is adopted and $f_{\pi}(=0.132 \mathrm{GeV})$ denotes the pion decay constant. The parameter $\mu_{\pi}$ in (10) is related to the chiral condensate

$$
\mu_{\pi}=\frac{m_{\pi}^{2}}{m_{u}+m_{d}}
$$

where $m_{\pi}$ is the pion mass while $m_{u}$ and $m_{d}$ denote current quark masses. In [5, 8, a value6 of $2 \mathrm{GeV}^{2}$ is taken for $\mu_{\pi}$. The contributions from transversely polarized photons which are of twist-3 accuracy, are parametrically suppressed by $\mu_{\pi} / Q$ as compared to the $\gamma_{L}^{*} \rightarrow \pi$ amplitudes (1). However, for values of $Q$ accessible in current experiments $\mu_{\pi} / Q$ is of order 1 .

The amplitude (10) is Fourier transformed to the impact parameter space (after partial fraction decomposition) and the integrand is multiplied by a Sudakov factor, exp $[-S]$, which represents gluon radiation in next-to-leading-log approximation using resummation techniques and having recourse to the renormalization group [15. The Sudakov factor cuts off the $b$-integration at $b_{0}=1 / \Lambda_{\mathrm{QCD}}$. In the modified perturbative approach the renormalization and factorization scales are $\mu_{R}=\max [\tau Q,(1-$ $\tau) Q, 1 / b]$ and $\mu_{F}=1 / b$, respectively. In [5, 8. the modified appoach is analogously applied to the $\gamma_{L}^{*} \rightarrow \pi$ amplitudes. The Sudakov factor guarantees the emergence of the twist-2 result for $Q^{2} \rightarrow \infty$.

\section{The pion pole}

A special feature of $\pi^{+}$production is the appearance of the pion pole. As has been shown in [16] the pion pole is part of the GPD $\tilde{E}$

$$
\tilde{E}_{\text {pole }}^{u}=-\tilde{E}_{\text {pole }}^{d}=\Theta(\xi-|x|) \frac{F_{P}(t)}{4 \xi} \Phi_{\pi}\left(\frac{x+\xi}{2 \xi}\right)
$$

where $F_{P}$ is the pole contribution to the pseudoscalar form factor of the nucleon which, with the help of PCAC and the Goldberger-Treiman relation, can be written as

$$
F_{P}(t)=\int_{-1}^{1} d x\left[\tilde{E}^{u}-\tilde{E}^{d}\right]=-2 m \frac{f_{\pi}}{F_{\pi}\left(Q^{2}\right)} \frac{\varrho_{\pi}}{t-m_{\pi}^{2}}
$$

4 This is the subprocess amplitude for $\pi^{+}$production. For the case of the $\pi^{0}$ the quark charges have to be taken out; they appear in the flavor combination of the GPDs.

5 It may seem appropriate to use an $l_{3}= \pm 1$ wave function for a particle moving along the 3-direction. Such a momentum space wave function is proportional to $k_{\perp}^{ \pm}=k_{\perp}^{1} \pm i k_{\perp}^{2}$ [14. However, its collinear reduction leads to the pseudotensor distribution amplitude; the pseudoscalar one is lacking in this wave function.

6 Taking the quark masses from [12], one finds $\mu_{\pi}=2.6_{-0.15}^{+0.52} \mathrm{GeV}$ while QCD sum rule analyses, e.g. [13], favor the value $1.6 \pm 0.2 \mathrm{GeV}$. 

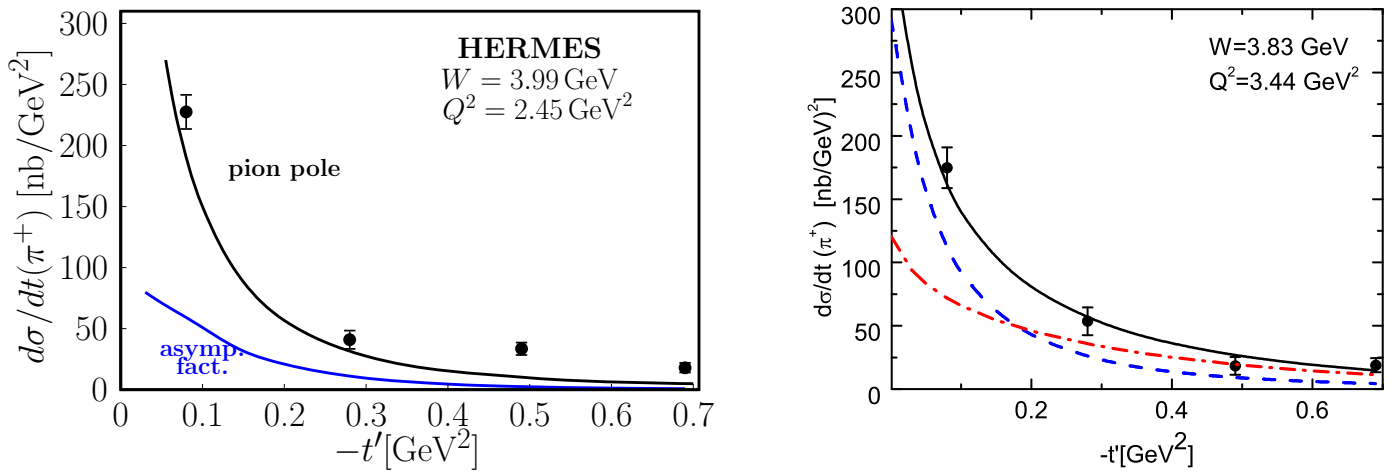

Fig. 3 The unseparated $\pi^{+}$cross section versus $-t^{\prime}$. The lines represent the pion pole contribution (16) with $F_{\pi}^{\text {pert }}$ replaced by the experimental value and the leading-twist result. Data are taken from [18].

Fig. 4 The unseparated $\pi^{+}$cross section. Data taken from [18. The solid (dashed, dash-dotted) curve represents the results of 8 for the unseparated (longitudinal, transverse) cross section.

with

$$
\varrho_{\pi}=\sqrt{2} g_{\pi N N} F_{\pi N N}(t) F_{\pi}\left(Q^{2}\right) .
$$

The coupling of the exchanged pion to the nucleons is described by the coupling constant $g_{\pi N N}(=$ $13.1 \pm 0.2)$ and a form factor parametrized as a monopole with a parameter $\Lambda_{N}(=0.44 \pm 0.07) ; F_{\pi}$ denotes the electromagnetic form factor of the pion. The convolution of $\tilde{E}_{\text {pole }}$ with the subprocess amplitude $\mathcal{H}_{0 \lambda, 0 \lambda}$ can be worked out analytically. The result leads to the following pole contribution to the longitudinal cross section

$$
\frac{d \sigma_{L}^{\text {pole }}}{d t}=4 \pi \frac{\alpha_{e m}}{\kappa} \frac{-t}{\left(t-m_{\pi}^{2}\right)^{2}} Q^{2} \varrho_{\pi}^{2} .
$$

However, in this calculation the pion form factor is only the leading-order perturbative contribution to it which is known to underestimate the experimental form factor [17 substantially, and consequently the $\pi^{+}$cross section, see Fig. 3. In [5,8] the perturbative result for $F_{\pi}$ was therefore replaced by its experimental value. This prescription is equivalent to computing the pion pole contribution as a oneparticle exchange 7 . With this replacement one sees that the pole term controls the $\pi^{+}$cross section at small $-t^{\prime}$, see Fig. 3. A detailed discussion of the pion pole contribution can be found in [19. It also plays a striking role in electroproduction of $\omega$ mesons [20].

\section{Phenomenology}

In order to make predictions and comparison with experiment the GPDs are needed. In [5, 8,11 , the GPDs are constructed with the help of the double-distribution representation. According to [21] a double distribution is parametrized as a product of a zero-skewness GPD and an appropriate weight function that generates the skewness dependence. The zero-skewness GPD itself is composed of its forward limit, $K(x, \xi=t=0)=k(x)$, multiplied by an exponential in $t$ with a profile function, $f(x)$, parametrized in a Regge-like manner

$$
f(x)=-\alpha^{\prime} \ln x+B
$$

at small $-t$. The GPD $\widetilde{H}$ at $\xi=0$ is taken from a recent analysis of the nucleon form factors [22] while $\tilde{E}$ is neglected. The forward limit of the transversity GPD $H_{T}$ is given by the transversity parton distributions, known from an analysis of the azimuthal asymmetry in semi-inclusive deep inelastic lepton-nucleon scattering and inclusive two-hadron production in electron-positron annihilations [23].It turns out, however, that this parametrization underestimates $H_{T}$. Its moments are about $40 \%$ smaller

\footnotetext{
7 For $Q^{2} \gg-t$ the virtuality of the exchanged pion can be neglected. The $\gamma^{*} \rightarrow \pi \pi^{*}$ vertex is therefore the pion form factor of the pion.
} 
than lattice QCD results [24] and it leads to a very deep dip in the forward $\pi^{0}$ cross section which is at variance with experiment 4]. Therefore, the normalization of $H_{T}$ is adjusted to the lattice QCD moments but the $x$-dependence of the transversity distributions is left unchanged. The forward limit of $\bar{E}_{T}$ is parametrized like the usual parton densities

$$
\bar{e}_{T}(x)=N x^{-\alpha}(1-x)^{\beta}
$$

with $\alpha=0.3$ for both $u$ and $d$ quarks and $\beta^{u}=4, \beta^{d}=5$. The normalization as well as the parameters $\alpha^{\prime}$ and $B$ for each of the transversity GPDs are estimated by fits to the HERMES data on the $\pi^{+}$ cross section [18] and to the lattice QCD results [25] on the moments of $\bar{E}_{T}$.

An example of the results for the $\pi^{+}$cross section is shown in Fig. 4, typical results for $\pi^{0}$ production in Fig. 1. At small $-t^{\prime}$ the $\pi^{+}$cross section is under control of the pion pole as discussed in Sect. 4. The contribution from $\widetilde{H}$ to the longitudinal cross section is minor. The transverse cross section, although suppressed by $\mu_{\pi}^{2} / Q^{2}$, is rather large and even dominates for $-t^{\prime} \gtrsim 0.2 \mathrm{GeV}^{2}$. It is governed by $H_{T}$, the contribution from $\bar{E}_{T}$ is very small. This fact can easily be understood considering the relative sign of $u$ and $d$ quark GPDs. For $H_{T}$ they have opposite signs but the same sign for $\bar{E}_{T}$. Moreover, $\bar{E}_{T}^{u}$ and $\bar{E}_{T}^{d}$ are of similar size 8 . Since the GPDs contribute to $\pi^{+}$production in the flavor combination

$$
K^{\pi^{+}}=K^{u}-K^{d}
$$

it is obvious that the contributions from $\bar{E}_{T}^{u}$ and $\bar{E}^{d}$ cancel each other to a large extent in contrast to those from $H_{T}$.

For $\pi^{0}$ production the situation is reversed since the GPDs appear now in the flavor combination

$$
K^{\pi^{0}}=\frac{1}{\sqrt{2}}\left(e_{u} K^{u}-e_{d} K^{d}\right) .
$$

Therefore, $\bar{E}_{T}^{u}$ and $\bar{E}_{T}^{d}$ add while there is a partial cancellation between $H_{T}^{u}$ and $H_{T}^{d}$. As can be seen from Eqs. (6) and (8) the $\bar{E}_{T}$-contribution is of the natural-parity type and, hence, makes up the transverse-transverse interference cross section (5), see Fig. 11. The transverse cross section, $d \sigma_{T} / d t$ receives contribution from both $H_{T}$ and $\bar{E}_{T}$. However, the sum

$$
\frac{d \sigma_{T}}{d t}+\frac{d \sigma_{T T}}{d t} \simeq \frac{1}{2 \kappa}\left|\mathcal{M}_{0-,++}\right|^{2}
$$

is only fed by $H_{T}$. According to [5, 8 ]

$$
\frac{d \sigma_{L}}{d t} \ll \frac{d \sigma_{T}}{d t} .
$$

Hence, to a good approximation the transverse cross section equals the unseparated one. The prediction (22) is consistent with the very small longitudinal-transverse interference cross section, see Fig. 1. It is to be emphasized that this prediction is what is expected in the limit $Q^{2} \rightarrow 0$ and not for $Q^{2} \rightarrow \infty$. With the approximation $d \sigma_{T} \simeq d \sigma$ one can directly extract the convolutions of $H_{T}^{\pi^{0}}$ and $\bar{E}_{T}^{\pi^{0}}$ from the data on the $\pi^{0}$ cross sections. Results for the convolutions are displayed in Fig. 5 at $Q^{2}=1.75 \mathrm{GeV}^{2}$ and $x_{B}=0.224$ and compared to the estimates made in [5,8]. There is a further test of the set of amplitudes (8): The 'constant' modulation of the asymmetry $A_{L L}$ measured with longitudinally polarized beam and target by the CLAS collaboration [30] for $\pi^{0}$ production, is related to the cross sections by

$$
\frac{A_{L L}^{\text {const }}}{\sqrt{1-\varepsilon}} \frac{d \sigma}{d t} \simeq \frac{d \sigma_{T}}{d t}+\frac{d \sigma_{T T}}{d t} \simeq \frac{d \sigma}{d t}+\frac{d \sigma_{T T}}{d t}
$$

( $\varepsilon$ denotes the ratio of the longitudinal and transversal photon fluxes). A violation of this relation would indicate contributions from other transversity GPDs, especially from $\widetilde{H}_{T}$ (see (7D)). In Fig. 6] the right and left hand sides of (23) are compared to each other. Within admittedly large errors there is agreement except, perhaps, at the largest values of $-t$. Of course small contributions from other transversity GPDs cannot be excluded.

8 In 26 it has been speculated that $\bar{E}_{T}$ is linearly related to the Boer-Mulders function. Indeed both the functions show the same pattern [27]. This pattern is also supported by large- $N_{c}$ considerations [28]. 

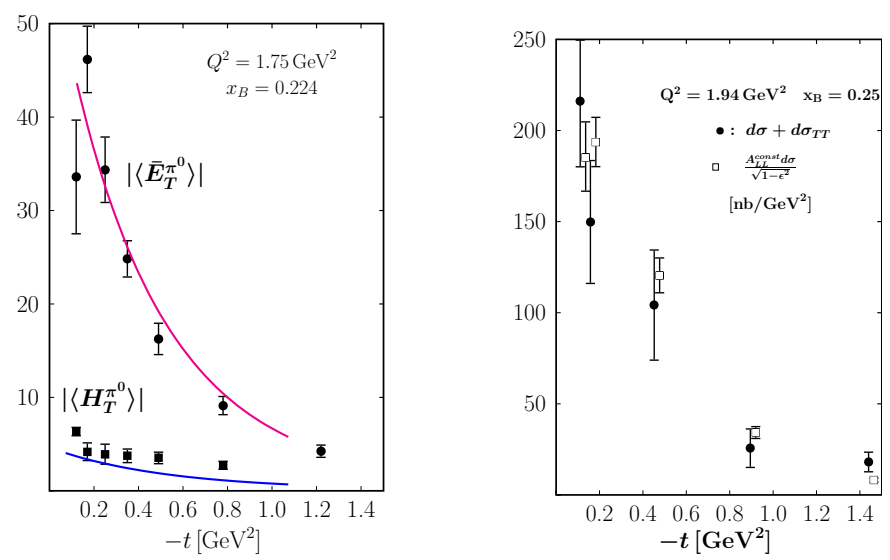

Fig. 5 The convolutions of $\bar{E}_{T}$ and $H_{T}$ extracted from the CLAS data on $\pi^{0}$ leptoproduction [4] and the estimates from [5,8].

Fig. 6 Testing the relation (23). Data taken from 4 and 30.

Data on $\pi^{0}$ production off neutrons as is planned to measure by the Jefferson Laboratory Hall A collaboration, will improve the flavor separation of the GPDs since they now appear in the combination

$$
K_{\text {neutron }}^{\pi^{0}}=\frac{1}{\sqrt{2}}\left(e_{u} K^{d}-e_{d} K^{u}\right) .
$$

In analogy to the proton case (see Fig. 6) one may extract the convolutions of $H_{T}$ and $\bar{E}_{T}$ from future data. According to the present estimate $\bar{E}_{T}$ for $u$ and $d$ quarks have about the same size. If this is correct the neutron/proton ratio of $d \sigma_{T T}$ is about one. On the other hand, for $d \sigma_{T}+d \sigma_{T T}$ the neutron/proton ratio is expected to be much smaller than one because of the properties of $H_{T}$.

The transversity GPDs play a similarly prominent role in leptoproduction of other pseudoscalar mesons. In Fig. 7 predictions for the $\eta$ cross sections are shown. Except in the proximity of the forward direction the unseparated cross section for $\eta$ production is considerably smaller than the $\pi^{0}$ one. In fact ratio $d \sigma(\eta) / d \sigma\left(\pi^{0}\right)$ amounts to about a third in fair agreement with the preliminary CLAS data on $\eta$ production 29. In the small $-t^{\prime}$ region where the GPD $H_{T}$ takes the lead, the $\eta / \pi^{0}$ ratio amounts to about 1 [5]. The behavior of the ratio found in [5] is in sharp contrast with earlier speculations 31 that the ratio is larger than 1 for all $t^{\prime}$. One can easily understand the results for the $\eta / \pi^{0}$ ratio by considering again the relative signs and sizes of the $u$ and $d$-quark GPDs. Under the plausible assumption $K^{s}=K^{\bar{s}}$ only the $u$ and $d$-quark GPDs in the combination 9

$$
K^{\eta} \simeq \frac{1}{\sqrt{6}}\left(e_{u} K^{u}+e_{d} K^{d}\right) .
$$

contribute to $\eta$ production. With regard to the different signs in (25) and (20) it is evident that $H_{T}$ plays a more important role for $\eta$ than for $\pi^{0}$ production while for $\bar{E}_{T}$ the situation is reversed with the consequence of a large $\eta / \pi^{0}$ ratio for $t^{\prime} \rightarrow 0$ and a small ratio otherwise. In the evaluation of the $\eta$ cross section $\eta-\eta^{\prime}$ mixing is to be taken into account [32. The $\eta$ cross sections are shown in Fig. 7. The transverse-transverse cross section is now very small because of the strong cancellation between $\bar{E}_{T}^{u}$ and $\bar{E}_{T}^{d}$.

The handbag approach can straightforwardly be generalized to the production of Kaons [5]. Some results on the cross sections for various pseudoscalar meson channels are shown in Fig. 8.

\section{Summary}

In this article the present status of the analysis of hard exclusive leptoproduction of pions and other pseudoscalar mesons within the handbag approach is reviewed. The present GPD parametrizations

\footnotetext{
9 Due to this assumption the flavor singlet and octet combinations are related by $K^{(1)}=\sqrt{2} K^{(8)}$.
} 

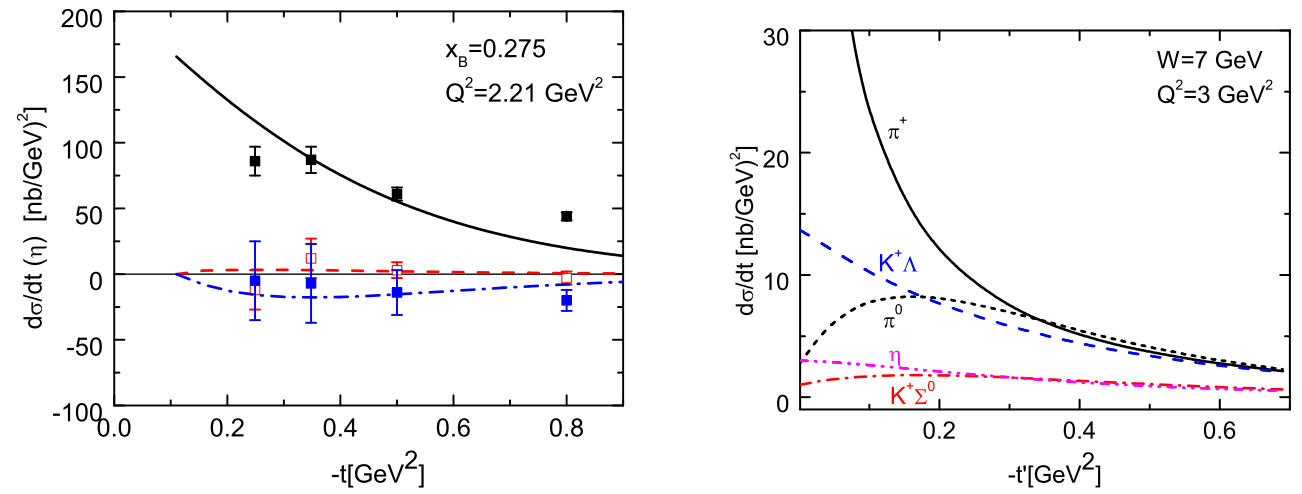

Fig. 7 The $\eta$ production cross sections. Predictions are from [5], preliminary data are from 29].

Fig. 8 Predictions for the unseparated cross sections of various pseudoscalar meson channels [5].

are to be considered as estimates which however reproduces the main features of the data. A detailed fit to all pion data is pending. The surprising result is the dominance of $\gamma_{T}^{*} \rightarrow \pi$ transitions. The leading-twist contribution is small, in particular for $\pi^{0}$ production. The ultimate justification of this observation would be a measurement of the unseparated cross sections. The JLab Hall A collaboration has done this for $\pi^{0}$ production. The experiment is under analysis.

The statement which has been mentioned in many papers and talks, that from pion leptoproduction we learn about the GPDs $\widetilde{H}$ and $\tilde{E}$ which was state of the art $10-15$ years ago, is to be revised: from pion leptoproduction we learn about contributions from transversely polarized photons and in particular about the transversity GPDs $H_{T}$ and $\bar{E}_{T}$.

\section{References}

1. A. V. Radyushkin, Phys. Lett. B 385, 333 (1996) hep-ph/9605431.

2. J. C. Collins, L. Frankfurt and M. Strikman, Phys. Rev. D 56, 2982 (1997) hep-ph/9611433.

3. A. Airapetian et al. [HERMES Collaboration], Phys. Lett. B 682, 345 (2010) arXiv:09907.2596 [hep-ex]].

4. I. Bedlinskiy et al. [CLAS Collaboration], Phys. Rev. C 90, no. 2, 025205 (2014) [Phys. Rev. C 90, no. 3, 039901 (2014)] arXiv:1405.0988 [nucl-ex]].

5. S. V. Goloskokov and P. Kroll, Eur. Phys. J. A 47, 112 (2011) arXiv:1106.4897 [hep-ph]].

6. P. Hoodbhoy and X. D. Ji, Phys. Rev. D 58. 054006 (1998) hep-ph/9801369.

7. M. Diehl, Eur. Phys. J. C 19, 485 (2001) hep-ph/0101335)

8. S. V. Goloskokov and P. Kroll, Eur. Phys. J. C 65, 137 (2010) arXiv:0906.0460 [hep-ph]].

9. S. Ahmad, G. R. Goldstein and S. Liuti, Phys. Rev. D 79, 054014 (2009) arXiv:0805.3568 [hep-ph]].

10. V. M. Braun and I. E. Filyanov, Z. Phys. C 48, 239 (1990) [Sov. J. Nucl. Phys. 52, 126 (1990)] [Yad. Fiz. 52, $199(1990)]$

11. S. V. Goloskokov and P. Kroll, Eur. Phys. J. C 53, 367 (2008) arXiv:0708.3569 [hep-ph]].

12. K. A. Olive et al. [Particle Data Group Collaboration], Chin. Phys. C 38, 090001 (2014).

13. P. Ball, JHEP 9901, 010 (1999) hep-ph/9812375.

14. X. d. Ji, J. P. Ma and F. Yuan, Eur. Phys. J. C 33, 75 (2004) hep-ph/0304107.

15. H. n. Li and G. F. Sterman, Nucl. Phys. B 381, 129 (1992).

16. L. Mankiewicz, G. Piller and A. Radyushkin, Eur. Phys. J. C 10, 307 (1999) hep-ph/9812467.

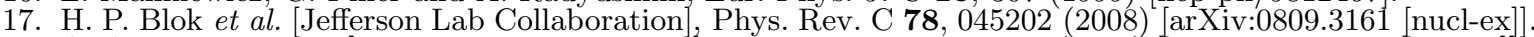

18. A. Airapetian et al. [HERMES Collaboration], Phys. Lett. B 682, 345 (2010) arXiv:0907.2596 [hep-ex]].

19. L. Favart, M. Guidal, T. Horn and P. Kroll, arXiv:1511.04535 [hep-ph].

20. S. V. Goloskokov and P. Kroll, Eur. Phys. J. A 50, no. 9, 146 (2014) arXiv:1407.1141 [hep-ph]].

21. I. V. Musatov and A. V. Radyushkin, Phys. Rev. D 61, 074027 (2000) hep-ph/9905376.

22. M. Diehl and P. Kroll, Eur. Phys. J. C 73, no. 4, 2397 (2013) arXiv:1302.4604 [hep-phi].

23. M. Anselmino, M. Boglione, U. D’Alesio, A. Kotzinian, F. Murgia, A. Prokudin and S. Melis, Nucl. Phys. Proc. Suppl. 191, 98 (2009) arXiv:0812.4366 [hep-ph]].

24. M. Gockeler et al. [QCDSF and UKQCD Collaborations], Phys. Lett. B 627, 113 (2005) hep-lat/0507001.

25. M. Gockeler et al. [QCDSF and UKQCD Collaborations], Phys. Rev. Lett. 98, 222001 (2007) hep-lat/0612032.

26. 'Mi. Burkardit ÁIP Conf. Proc 915, 313 (2007) hep-ph/0611256

27. V. Barone, S. Melis and A. Prokudin, Phys. Rev. D 81, 114026 (2010) arXiv:0912.5194 [hep-ph]].

28. P. Schweitzer and C. Weiss, arXiv:1601.03016 [hep-ph].

29. V. Kubarovsky [for the CLAS Collaboration], arXiv:1601.04367 [hep-ex]

30. A. Kim et al., arXiv:1511.03338 [nucl-ex].

31. M. I. Eides, L. L. Frankfurt and M. I. Strikman, Phys. Rev. D 59, 114025 (1999) hep-ph/9809277.

32. T. Feldmann, P. Kroll and B. Stech, Phys. Rev. D 58, 114006 (1998) hep-ph/980z40̄y 